\title{
Exact solutions and multi-symplectic structure of the generalized KdV-type equation
}

\author{
Xiao-Feng Yang ${ }^{1 *}$, Zi-Chen Deng ${ }^{2}$, Qing-Jun $\mathrm{Li}^{2}$ and Yi Wei ${ }^{1}$
}

\section{"Correspondence:}

yangxiaofeng@nwsuaf.edu.cn

'Department of Applied

Mathematics, Northwestern

Polytechnical University, Xi'an, 710072, P.R. China

Full list of author information is available at the end of the article

\section{Springer}

\begin{abstract}
The homogeneous balance of undetermined coefficients method is proposed to obtain not only exact solutions but also multi-symplectic structure of some nonlinear partial differential equations. Bilinear equation, $\mathrm{N}$-soliton solutions, traveling wave solutions and multi-symplectic structure are obtained by applying the proposed method to the KdV equation. Accordingly, the definition and multi-symplectic structure of the generalized KdV-type equation are given. The proposed method is also a standard and computable method, which can be generalized to deal with some types of nonlinear partial differential equations.
\end{abstract}

MSC: 35Q55; 35Q80; 35G25

Keywords: homogeneous balance of undetermined coefficients method; multi-symplectic structure; generalized KdV-type equation; bilinear equation; $\mathrm{N}$-soliton solution; traveling wave solution

\section{Introduction}

Nonlinear partial differential equations (NLPDEs) are used to describe a variety of phenomena not only in physics, but also in several other fields.

The way to obtain the exact solutions of NLPDEs should be considered firstly for any given NLPDEs. There are numerous powerful methods, such as the inverse scattering method [1], the homotopy perturbation method [2], the first integral method [3], the $\left(\frac{G^{\prime}}{G}\right)$-expansion method $[4,5]$, Hirota's method $[6,7]$, the homogeneous balance method $[8,9]$, the variational iteration method [10], the tanh-sech method [11], the modified simple equation method [12], which can be used to construct the exact solutions of NLPDEs.

As is well known, for most of NLPDEs, it is difficult to obtain the exact solutions or there is no exact solution. In these cases, it is natural to resort to the numerical methods. Analogous to the analytical methods, there are many numerical methods to solve NLPDEs. However, considering the stability and effectiveness of numerical algorithms, not all numerical methods can be used to solve NLPDEs [13]. Based on the basic rule that all numerical methods should preserve the intrinsic properties of NLPDEs as much as possible, a multi-symplectic algorithm for Hamiltonian PDEs was presented by Marsden et al. [14] who derived a numerical scheme from the Lagrangian formulation in firstorder field using a discrete variational principle. Bridges and Reich $[15,16]$ proposed

(c) 2015 Yang et al. This article is distributed under the terms of the Creative Commons Attribution 4.0 International License (http://creativecommons.org/licenses/by/4.0/), which permits unrestricted use, distribution, and reproduction in any medium, provided you give appropriate credit to the original author(s) and the source, provide a link to the Creative Commons license, and indicate if changes were made. 
multi-symplectic algorithms from Hamiltonian formalism. Bridges, Reich and Moore et al. proposed multi-symplectic Runge-Kutta collocation scheme [17], Euler box scheme, Preissmen box scheme, explicit midpoint scheme, spectral discretization scheme for some Hamiltonian PDEs [15-18]. Wang and Chen et al. developed multi-symplectic algorithms $[13,19,20]$. Hu et al. proposed generalized multi-symplectic algorithms [21-24].

The above multi-symplectic algorithms for NLPDEs have been developed well. Longtime numerical stability, high precision and preserving the intrinsic properties of NLPDEs have been proved. Generally, for given NLPDEs, how to construct multi-symplectic structure of the NLPDEs is an important issue and a step to solve NLPDEs. Moreover, to our knowledge, there are few methods which consider not only the exact solutions but also the numerical solutions for given NLPDEs.

Based on these problems, a new method, which is called the homogeneous balance of undetermined coefficients method, is used to construct not only the exact solutions but also multi-symplectic structure for given NLPDEs.

To illustrate the validity of the proposed method, let us consider the celebrated KdV equation in the form

$$
u_{t}+u u_{x}+\delta u_{x x x}=0
$$

where $\delta$ is a constant.

Firstly, the homogeneous balance of undetermined coefficients method is used to obtain $N$-soliton solutions and traveling wave solutions of Eq. (1). Secondly, we will construct multi-symplectic structure of Eq. (1). Thirdly, we will consider NLPDE

$$
u_{t}+f(u) u_{x}+\left(g\left(u_{x}\right)\right)_{x x}=0
$$

which is called generalized KdV-type equation with $f$ and $g$ being smooth functions. Finally, similar to constructed multi-symplectic structure of Eq. (1), the multi-symplectic structure of Eq. (2) is given.

The remainder of this paper is organized as follows: the homogeneous balance of undetermined coefficients method is described in Section 2. In Section 3, the proposed method is used to obtain $N$-soliton solutions and traveling wave solutions of Eq. (1). In Section 4, multi-symplectic structure of the KdV equation is given by the proposed method. In Section 5, the definition of generalized KdV-type equation is given. Moreover, we construct multi-symplectic structure of the generalized KdV-type equation. In Section 6, some conclusions are given.

\section{Description of the homogeneous balance of undetermined coefficients method}

Let us consider a general NLPDE, say, in two variables

$$
P\left(u, u_{t}, u_{x}, u_{x x}, u_{x t}, \ldots\right)=0 \text {, }
$$

where $P$ is a polynomial function of its arguments, the subscripts denote the partial derivatives. The homogeneous balance of undetermined coefficients method consists of three steps. 
Step 1. Suppose that the solution of Eq. (3) is of the form

$$
u=a_{m n}(\ln w)_{m, n}+\sum_{\substack{i, j=0 \\ i+j \neq 0, m+n}}^{i=m, j=n} a_{i j}(\ln w)_{i, j}+a_{00}
$$

where $u=u(x, t), w=w(x, t),(\ln w)_{i, j}=\frac{\partial^{i+j}(\ln w(x, t))}{\partial x^{i} \partial t^{j}}, m, n$ (balance numbers) and $a_{i j}(i=$ $0,1, \ldots, m ; j=0,1, \ldots, n)$ (balance coefficients) are constants to be determined later.

By balancing the highest nonlinear terms and the highest order partial derivative terms, balance numbers are obtained. Substituting Eq. (4) into Eq. (3) and balancing the terms with $\left(\frac{w_{x}}{w}\right)^{i}\left(\frac{w_{t}}{w}\right)^{j}$ yield a set of algebraic equations for balance coefficients.

Step 2. Solving the set of algebraic equations and simplifying Eq. (3), we can get the bilinear equation or homogeneous equation of Eq. (3) directly or after integrating some times (generally, integrating times equals the orders of lowest partial derivative of Eq. (3)) with respect to $x, t$.

Step 3. Generally, in order to obtain the exact solutions of Eq. (3), there are two schemes to deal with the bilinear equation or homogeneous equation of Eq. (3).

(I) Applying the recursive method to the bilinear equation or homogeneous equation of Eq. (3), $N$-soliton solutions of Eq. (3) can be obtained.

(II) By using traveling wave transformations

$$
w(x, t)=w(\xi), \quad \xi=x-V t,
$$

the bilinear equation or homogeneous equation of Eq. (3) satisfies the following ODE:

$$
w^{\prime \prime}+\lambda w^{\prime}+\mu w=0
$$

where the prime denotes the derivation with respect to $\xi$ and $\lambda, \mu$ and $V$ are constants to be determined later.

Substituting Eqs. (5) and (6) into the bilinear equation or homogeneous equation of Eq. (3), it is converted into the following equation:

$$
l_{1} w^{2}+l_{2} w w^{\prime}+l_{3} w^{\prime 2}=0
$$

where $l_{1}, l_{2}$ and $l_{3}$ are polynomial functions of $V, \lambda, \mu$.

Setting $l_{1}=l_{2}=l_{3}=0$ yields a set of algebraic equations for $V, \lambda, \mu$. Solving the set of algebraic equations and using the solutions of Eq. (6), $w$ can be determined. Substituting $w$ into Eq. (4), exact traveling wave solutions of Eq. (3) are obtained.

Next, we choose Eq. (1), namely the KdV equation, to illustrate our method.

\section{Application to the KdV equation}

In this section, the method proposed in Section 2 is used to obtain $N$-soliton solutions and traveling wave solutions of the KdV equation.

Suppose that the solution of Eq. (1) is in the form of Eq. (4). Balancing $u_{x x x}$ and $u u_{x}$ in Eq. (1), it is required that $m+3=2 m+1, n=2 n$. Then Eq. (4) can be written as

$$
u=a_{20}(\ln w)_{x x}+a_{10}(\ln w)_{x}+a_{00}
$$

where $a_{i 0}(i=0,1,2)$ are constants to be determined later. 
Substituting Eq. (8) into Eq. (1) and equating the coefficients of $\left(\frac{w_{x}}{w}\right)^{5}$ and $\left(\frac{w_{x}}{w}\right)^{4}$ on the left-hand side of Eq. (1) to zero yield a set of algebraic equations for $a_{20}$ and $a_{10}$ as follows:

$$
-2 a_{20}^{2}+24 \delta a_{20}=0, \quad 3 a_{20} a_{10}-6 \delta a_{10}=0 .
$$

Solving the above algebraic equations, we get $a_{20}=12 \delta, a_{10}=0$. Substituting $a_{20}$ and $a_{10}$ back into Eq. (8), we get

$$
u=12 \delta(\ln w)_{x x}+a_{00}
$$

where $a_{00}$ is an arbitrary constant.

Substituting Eq. (9) into Eq. (1), we get

$$
12 \delta\left(k_{1}+k_{2}+k_{3}\right)=0
$$

where

$$
\begin{aligned}
& k_{1}=\frac{w_{x x t}}{w}-\frac{2 w_{x} w_{x t}+w_{x x} w_{t}}{w^{2}}+\frac{2 w_{x}^{2} w_{t}}{w^{3}}, \quad k_{2}=a_{00}\left(\frac{w_{x x x}}{w}-\frac{3 w_{x x} w_{x}}{w^{2}}+\frac{2 w_{x}^{3}}{w^{3}}\right), \\
& k_{3}=\delta\left(\frac{w_{x x x x x}}{w}+\frac{2 w_{x x x} w_{x x}-5 w_{x x x x} w_{x}}{w^{2}}+\frac{16 w_{x x x} w_{x}^{2}-6 w_{x} w_{x x}^{2}}{w^{3}}\right) .
\end{aligned}
$$

Simplifying Eq. (10) and integrating with respect to $x$ once, we get

$$
\frac{\partial}{\partial x}\left(\frac{\left(w_{x t} w-w_{x} w_{t}\right)+\delta\left(w_{x x x x} w-4 w_{x} w_{x x x}+3 w_{x x}^{2}\right)+a_{00}\left(w_{x x} w-w_{x}^{2}\right)}{w^{2}}\right)=0
$$

Equation (11) is identical to

$$
\left(w_{x t} w-w_{x} w_{t}\right)+\delta\left(w_{x x x x} w-4 w_{x} w_{x x x}+3 w_{x x}^{2}\right)+a_{00}\left(w_{x x} w-w_{x}^{2}\right)-C(t) w^{2}=0
$$

where $C(t)$ is an arbitrary function of $t$.

Especially, taking $C(t)$ as zero in Eq. (12), we get the bilinear equation of Eq. (1)

$$
\left(w_{x t} w-w_{x} w_{t}\right)+\delta\left(w_{x x x x} w-4 w_{x} w_{x x x}+3 w_{x x}^{2}\right)+a_{00}\left(w_{x x} w-w_{x}^{2}\right)=0
$$

Equation (13) can be written concisely in terms of $D$-operator as

$$
\left(D_{x} D_{t}+\delta D_{x}^{4}+a_{00} D_{x}^{2}\right) w \cdot w=0
$$

where

$$
D_{x}^{m} D_{t}^{n} a \cdot b=\left.\left(\partial_{x}-\partial_{x^{\prime}}\right)^{m}\left(\partial_{t}-\partial_{t^{\prime}}\right)^{n} a(x, t) b\left(x^{\prime}, t^{\prime}\right)\right|_{x^{\prime}=x, t^{\prime}=t} .
$$

Remark 1 Applying Hirota's method [7] to Eq. (1), the bilinear equation of Eq. (1) can be written as

$$
\left(D_{x} D_{t}+\delta D_{x}^{4}\right) w \cdot w=0
$$


Equation (15) is obtained by setting $a_{00}=0$ in Eq. (14). Obviously, Eq. (15) is a special case of Eq. (14).

(I) Now, by using the bilinear and recursive properties of Eq. (13), $N$-soliton solutions of Eq. (1) can be obtained.

Equation (13) can be written as

$$
\mathbf{X}^{\mathrm{T}} \mathbf{B X}+\mathbf{Y}^{\mathrm{T}} \mathbf{F Y}=0
$$

where $\mathbf{X}=\left(w, w_{x}, w_{t}, w_{x t}\right)^{\mathrm{T}}, \mathbf{Y}=\left(w, w_{x}, w_{x x}, w_{x x x}, w_{x x x x}\right)^{\mathrm{T}}$ and

$$
\mathbf{B}=\left(\begin{array}{cccc}
0 & 0 & 0 & \frac{1}{2} \\
0 & 0 & -\frac{1}{2} & 0 \\
0 & -\frac{1}{2} & 0 & 0 \\
\frac{1}{2} & 0 & 0 & 0
\end{array}\right), \quad \mathbf{F}=\left(\begin{array}{ccccc}
0 & 0 & \frac{a_{00}}{2} & 0 & \frac{\delta}{2} \\
0 & -a_{00} & 0 & -2 \delta & 0 \\
\frac{a_{00}}{2} & 0 & 3 \delta & 0 & 0 \\
0 & -2 \delta & 0 & 0 & 0 \\
\frac{\delta}{2} & 0 & 0 & 0 & 0
\end{array}\right) .
$$

Obviously, $h_{i}=e^{P_{i} x-\left(a_{00} P_{i}+\delta P_{i}^{3}\right) t+\xi_{i}^{0}}(i=1,2, \ldots)\left(P_{i}\right.$ and $\xi_{i}^{0}$ are arbitrary constants $)$ are solutions of Eq. (16). Suppose that $h_{i} \neq h_{j}(i \neq j ; i, j=1,2, \ldots)$. Setting

$$
w_{1}=1+h_{1}
$$

it is easy to find that $w_{1}$ is a solution of Eq. (16).

Substituting $w_{2}=w_{1}+h_{2}\left(1, h_{1}\right)\left(1, b_{12}\right)^{\mathrm{T}}$ ( $b_{12}$ is a constant to be determined) into Eq. (16) and using linear independence of functions $1, h_{1}, h_{2}, h_{1} h_{2}$, we get

$$
w_{2}=1+h_{1}+h_{2}+b_{12} h_{1} h_{2}
$$

where $b_{12}=\frac{\left(P_{1}-P_{2}\right)^{2}}{\left(P_{1}+P_{2}\right)^{2}}$.

Substituting $w_{3}=w_{2}+h_{3}\left(1, h_{1}, h_{2}, h_{1} h_{2}\right)\left(1, b_{13}, b_{23}, b_{123}\right)^{\mathrm{T}}\left(b_{13}, b_{23}\right.$ and $b_{123}$ are constants to be determined) into Eq. (16) and using linear independence of functions $1, h_{1}, h_{2}, h_{3}$, $h_{1} h_{2}, h_{1} h_{3}, h_{2} h_{3}, h_{1} h_{2} h_{3}$, we get

$$
w_{3}=1+h_{1}+h_{2}+b_{12} h_{1} h_{2}+b_{13} h_{1} h_{3}+b_{23} h_{2} h_{3}+b_{123} h_{1} h_{2} h_{3},
$$

where

$$
\begin{aligned}
& b_{13}=\frac{\left(P_{1}-P_{3}\right)^{2}}{\left(P_{1}+P_{3}\right)^{2}}, \\
& b_{23}=\frac{\left(P_{2}-P_{3}\right)^{2}}{\left(P_{2}+P_{3}\right)^{2}}, \\
& b_{123}=b_{12} b_{13} b_{23}=\frac{\left(P_{1}-P_{2}\right)^{2}\left(P_{1}-P_{3}\right)^{2}\left(P_{2}-P_{3}\right)^{2}}{\left(P_{1}+P_{2}\right)^{2}\left(P_{1}+P_{3}\right)^{2}\left(P_{2}+P_{3}\right)^{2}} .
\end{aligned}
$$

Similarly, $w_{N}$ can be obtained by recursiveness.

Substituting $w_{1}, w_{2}, \ldots, w_{N}$ into Eq. (9), $N$-soliton solution of Eq. (1) can be obtained. 
Remark $2 \mathrm{~N}$-soliton solution of Eq. (1) can be obtained by applying the perturbation method to Eq. (13). Suppose that $w$ can be expanded as follows:

$$
w=1+\varepsilon c_{1}+\varepsilon^{2} c_{2}+\cdots+\varepsilon^{N} c_{N}+\cdots,
$$

where $\varepsilon$ is a parameter and $c_{i}=c_{i}(x, t)(i=1,2, \ldots)$.

Substituting Eq. (20) into Eq. (14) and arranging it at each order of $\varepsilon$, we get

$$
\begin{aligned}
& \varepsilon: D_{x}\left(D_{t}+a_{00} D_{x}+\delta D_{x}^{3}\right)\left(c_{1} \cdot 1+1 \cdot c_{1}\right)=0, \\
& \varepsilon^{2}: D_{x}\left(D_{t}+a_{00} D_{x}+\delta D_{x}^{3}\right)\left(c_{2} \cdot 1+c_{1} \cdot c_{1}+1 \cdot c_{2}\right)=0, \\
& \varepsilon^{3}: D_{x}\left(D_{t}+a_{00} D_{x}+\delta D_{x}^{3}\right)\left(c_{3} \cdot 1+c_{2} \cdot c_{1}+c_{1} \cdot c_{2}+1 \cdot c_{3}\right)=0,
\end{aligned}
$$

The order- $\varepsilon$ equation can be rewritten as a linear differential equation for $c_{1}$ as follows:

$$
\frac{\partial}{\partial x}\left(\frac{\partial}{\partial t}+a_{00} \frac{\partial}{\partial x}+\delta \frac{\partial^{3}}{\partial x^{3}}\right) c_{1}=0
$$

Solving Eq. (21), we get

$$
c_{1}=e^{P_{1} x-\left(a_{00} P_{1}+\delta P_{1}^{3}\right) t+\xi_{i}^{0}},
$$

where $P_{1}$ and $\xi_{1}^{0}$ are arbitrary constants.

The coefficient of $\varepsilon^{2}$ can be rearranged as follows:

$$
2 \frac{\partial}{\partial x}\left(\frac{\partial}{\partial t}+a_{00} \frac{\partial}{\partial x}+\delta \frac{\partial^{3}}{\partial x^{3}}\right) c_{2}=-D_{x}\left(D_{t}+a_{00} D_{x}+\delta D_{x}^{3}\right) c_{1} \cdot c_{1} .
$$

Substituting Eq. (22) into Eq. (23), the right-hand side of Eq. (23) equals zero. Therefore, we can choose

$$
c_{2}=0 .
$$

Substituting Eqs. (22) and (24) into Eq. (20), we get

$$
w_{1}=1+e^{P_{1} x-\left(a_{00} P_{1}+\delta P_{1}^{3}\right) t+\xi_{1}^{0}},
$$

where $P_{1}$ and $\xi_{1}^{0}$ are arbitrary constants.

If we choose $w_{1}=e^{P_{1} x-\left(a_{00} P_{1}+\delta P_{1}^{3}\right) t}+e^{P_{2} x-\left(a_{00} P_{2}+\delta P_{2}^{3}\right) t}$ in Eq. (21), similar to the above process, we can get

$$
w_{2}=1+e^{\eta_{1}}+e^{\eta_{2}}+\frac{\left(P_{1}-P_{2}\right)^{2}}{\left(P_{1}+P_{2}\right)^{2}} e^{\eta_{1}+\eta_{2}},
$$

where $\eta_{i}=P_{i} x-\left(a_{00} P_{i}+\delta P_{i}^{3}\right) t+\xi_{i}^{0}, P_{i}, \xi_{i}^{0}(i=1,2)$ are arbitrary constants.

Substituting Eqs. (25) and (26) into Eq. (9), 1-soliton and 2-soliton solutions of Eq. (1) can be obtained respectively.

Similarly, $N$-soliton solution of Eq. (1) can be obtained. 
Comparing our method with the perturbation method, our method is simpler than the perturbation method because of recursiveness.

Remark 3 Obviously, setting $a_{00}=0$ in Eqs. (17), (18) and (19), 1-soliton, 2-soliton and 3-soliton solutions of Eq. (13) are identical to Hirota's results [7].

Remark 4 By using the properties of $D$-operator [7], a Bäcklund transformation of Eq. (14) can be obtained as follows:

$$
\begin{aligned}
& \left(D_{t}+\left(a_{00}+\alpha\right) D_{x}+\delta D_{x}^{3}\right) w^{*} \cdot w=0, \\
& \left(D_{x}^{2}-\beta D_{x}-\alpha\right) w^{*} \cdot w=0
\end{aligned}
$$

where $w^{*}$ and $w$ satisfy Eq. (14), and $\alpha, \beta$ and $a_{00}$ are arbitrary constants.

(II) Now, we discuss the traveling wave solutions of Eq. (1) by using traveling wave transformations.

Using transformations $w(x, t)=w(\xi), \xi=x-V t$, Eq. (13) is reduced to

$$
\left(a_{00}-V\right)\left(w^{\prime \prime} w-w^{\prime 2}\right)+\delta\left(w^{\prime \prime \prime \prime} w-4 w^{\prime} w^{\prime \prime \prime}+3 w^{\prime \prime 2}\right)=0
$$

or

$$
\mathbf{Y}^{* \mathrm{~T}} \mathbf{F}^{*} \mathbf{Y}^{*}=0
$$

where $\mathbf{Y}^{*}=\left(w, w^{\prime}, w^{\prime \prime}, w^{\prime \prime \prime}, w^{\prime \prime \prime \prime}\right)^{\mathrm{T}}$, the prime denotes the derivation with respect to $\xi$, and $V$ is a constant to be determined later, and

$$
\mathbf{F}^{*}=\left(\begin{array}{ccccc}
0 & 0 & \frac{a_{00}-V}{2} & 0 & \frac{\delta}{2} \\
0 & V-a_{00} & 0 & -2 \delta & 0 \\
\frac{a_{00}-V}{2} & 0 & 3 \delta & 0 & 0 \\
0 & -2 \delta & 0 & 0 & 0 \\
\frac{\delta}{2} & 0 & 0 & 0 & 0
\end{array}\right) .
$$

Noticing the bilinear property of Eqs. (27a) and (27b), $w$ can satisfy the following ODE:

$$
w^{\prime \prime}+\lambda w^{\prime}+\mu w=0
$$

where $\lambda$ and $\mu$ are parameters.

Substituting Eq. (28) into Eqs. (27a) and (27b), we get

$$
l_{1} w^{2}+l_{2} w w^{\prime}+l_{3} w^{\prime 2}=0
$$

where

$$
\begin{aligned}
& l_{1}=\mu\left(V-a_{00}+\delta\left(4 \mu-\lambda^{2}\right)\right), \quad l_{2}=\lambda\left(V-a_{00}+\delta\left(4 \mu-\lambda^{2}\right)\right), \\
& l_{3}=V-a_{00}+\delta\left(4 \mu-\lambda^{2}\right) .
\end{aligned}
$$


Setting $l_{1}=l_{2}=l_{3}=0$ yields a set of algebraic equations for $V, \lambda, \mu$. Solving this set of algebraic equations, we get

$$
V=a_{00}+\delta\left(\lambda^{2}-4 \mu\right)
$$

where $\lambda, \mu$ and $a_{00}$ are arbitrary constants. Substituting Eq. (28) into Eq. (9), we get

$$
u=-12 \delta\left(\frac{w^{\prime}}{w}+\frac{\lambda}{2}\right)^{2}+3 \delta\left(\lambda^{2}-4 \mu\right)+a_{00}
$$

Substituting the general solutions of Eq. (28) into Eq. (31), we get three types of traveling wave solutions of Eq. (1) as follows.

When $\lambda^{2}-4 \mu>0$,

$$
u_{1}(x, t)=u_{1}(\xi)=-3 \delta A\left(\frac{C_{1} e^{\frac{\sqrt{A}}{2} \xi}-C_{2} e^{-\frac{\sqrt{A}}{2} \xi}}{C_{1} e^{\frac{\sqrt{A}}{2} \xi}+C_{2} e^{-\frac{\sqrt{A}}{2} \xi}}\right)^{2}+3 \delta A+a_{00},
$$

where

$$
A=\lambda^{2}-4 \mu, \quad V=a_{00}+A \delta, \quad \xi=x-\left(a_{00}+A \delta\right) t,
$$

$\lambda, \mu, C_{1}, C_{2}$ and $a_{00}$ are arbitrary constants.

Taking $C_{1}=\frac{C_{3}+C_{4}}{2}$ and $C_{2}=\frac{C_{3}-C_{4}}{2}$, Eq. (32) can be rewritten as

$$
u_{2}(x, t)=u_{2}(\xi)=-3 \delta A\left(\frac{C_{3} \sinh \frac{\sqrt{A}}{2} \xi+C_{4} \cosh \frac{\sqrt{A}}{2} \xi}{C_{3} \cosh \frac{\sqrt{A}}{2} \xi+C_{4} \sinh \frac{\sqrt{A}}{2} \xi}\right)^{2}+3 \delta A+a_{00},
$$

where $C_{3}, C_{4}$ and $a_{00}$ are arbitrary constants, $A, V$ and $\xi$ are given by Eq. (33).

Especially, if $\left|\frac{C_{4}}{C_{3}}\right|<1$, then Eq. (34) is reduced to

$$
u_{3}(x, t)=u_{3}(\xi)=3 \delta A \operatorname{sech}^{2}\left(\frac{\sqrt{A}}{2} \xi+\xi_{0}\right)+a_{00}
$$

where $C_{3}, C_{4}$ and $a_{00}$ are arbitrary constants, $A, V$ and $\xi$ are given by Eq. (33), $\xi_{0}=$ $\operatorname{arctanh} \frac{C_{4}}{C_{3}}$.

When $\lambda^{2}-4 \mu<0$,

$$
u_{4}(x, t)=u_{4}(\xi)=3 \delta A\left(\frac{-C_{1} \sin \frac{\sqrt{-A}}{2} \xi+C_{2} \cos \frac{\sqrt{-A}}{2} \xi}{C_{1} \cos \frac{\sqrt{-A}}{2} \xi+C_{2} \sin \frac{\sqrt{-A}}{2} \xi}\right)^{2}+3 \delta A+a_{00},
$$

where $C_{1}, C_{2}$ and $a_{00}$ are arbitrary constants, $A, V$ and $\xi$ are given by Eq. (33).

Obviously, Eq. (36) can be written as

$$
u_{5}(x, t)=u_{5}(\xi)=3 \delta A \sec ^{2}\left(\frac{\sqrt{-A}}{2} \xi+\xi_{0}\right)+a_{00}
$$

where $C_{1}, C_{2}$ and $a_{00}$ are arbitrary constants, $A, V$ and $\xi$ are given by Eq. (33), $\xi_{0}=$ $-\arctan \frac{C_{2}}{C_{1}}$. 
When $\lambda^{2}-4 \mu=0$,

$$
u_{6}(x, t)=u_{6}(\xi)=-12 \delta\left(\frac{C_{2}}{C_{1}+C_{2} \xi}\right)^{2}+a_{00}
$$

where $V=a_{00}, \xi=x-a_{00} t, C_{1}, C_{2}$ and $a_{00}$ are arbitrary constants.

Comparing with the $\left(\frac{G^{\prime}}{G}\right)$-expansion method, $u_{i}(x, t)(i=1, \ldots, 6)$ are identical to the results of using the $\left(\frac{G^{\prime}}{G}\right)$-expansion method. Our method is simpler than the $\left(\frac{G^{\prime}}{G}\right)$-expansion method because our method preserves the intrinsic properties (e.g., symmetry and bilinearity) of the $\mathrm{KdV}$ equation. Moreover, $\mathrm{N}$-soliton solution of the $\mathrm{KdV}$ cannot be obtained by the $\left(\frac{G^{\prime}}{G}\right)$-expansion method because traveling wave transformation is used somewhat early.

\section{Multi-symplectic structure of the KdV equation}

In this section, multi-symplectic structure of the $K d V$ equation is given by using the results of Section 2. Firstly, we quote the definition of multi-symplectic structure which is given by Bridges and Reich [15].

Definition 1 [15] Let $\mathbf{M}$ and $\mathbf{K}$ be any skew-symmetric matrices on $R^{n}(n \geq 3)$ and let $S$ : $R^{n} \rightarrow R$ be any smooth function. A system of the following form is a Hamiltonian system on a multi-symplectic structure:

$$
\mathbf{M Z}_{t}+\mathbf{K Z}_{x}=\nabla_{\mathbf{Z}} S(\mathbf{Z}), \quad \mathbf{Z} \in R^{n},(x, t) \in R^{2}
$$

where the gradient $\nabla_{\mathbf{Z}}$ is defined with respect to the standard inner product on $R^{n}$, denoted by $\langle\cdot, \cdot\rangle$.

Given a PDE, how to determine Hamiltonian function $S$ and state variable $\mathbf{Z}$ is key of constructing multi-symplectic structure.

Consider Eq. (1), setting $a_{00}=0$ in Eq. (9) (see Section 2) and substituting it into Eq. (1) yield

$$
(\ln w)_{x x t}+12 \delta(\ln w)_{x x}(\ln w)_{x x x}+\delta(\ln w)_{x x x x x}=0
$$

Integrating the above equation with respect to $x$ once and setting integration constant to zero yield

$$
(\ln w)_{x t}+6 \delta\left((\ln w)_{x x}\right)^{2}+\delta(\ln w)_{x x x x}=0
$$

Introducing a state variable $\mathbf{Z}^{*}=\left((\ln w)_{x},(\ln w)_{x x},(\ln w)_{x x x},(\ln w)_{x t}\right)^{\mathrm{T}}$, we have

$$
\mathbf{M}_{1} \mathbf{Z}_{t}^{*}+\mathbf{K}_{1} \mathbf{Z}_{x}^{*}=\mathbf{H}_{1}
$$

where

$$
\mathbf{M}_{1}=\left(\begin{array}{cccc}
0 & 0 & 0 & 0 \\
0 & 0 & 0 & 0 \\
0 & 0 & 0 & 0 \\
0 & 0 & 0 & 0
\end{array}\right), \quad \mathbf{K}_{1}=\left(\begin{array}{cccc}
0 & 0 & 0 & 0 \\
0 & 0 & -2 \delta & 0 \\
0 & 0 & 0 & 0 \\
0 & 0 & 0 & 0
\end{array}\right),
$$




$$
\mathbf{H}_{1}=\left(\begin{array}{c}
0 \\
12 \delta\left((\ln w)_{x x}\right)^{2}+2(\ln w)_{x t} \\
0 \\
0
\end{array}\right) .
$$

Noticing antisymmetry of state variable's coefficient matrix, we have

$$
\mathbf{M}_{2} \mathbf{Z}_{t}^{*}+\mathbf{K}_{2} \mathbf{Z}_{x}^{*}=\mathbf{H}_{2} \text {, }
$$

where

$$
\begin{aligned}
\mathbf{M}_{2} & =\left(\begin{array}{cccc}
0 & 0 & 0 & 0 \\
0 & 0 & 0 & 0 \\
0 & 0 & 0 & 0 \\
0 & 0 & 0 & 0
\end{array}\right), \quad \mathbf{K}_{2}=\left(\begin{array}{cccc}
0 & 0 & 0 & 0 \\
0 & 0 & -2 \delta & 0 \\
0 & 2 \delta & 0 & 0 \\
0 & 0 & 0 & 0
\end{array}\right), \\
\mathbf{H}_{2} & =\left(\begin{array}{c}
12 \delta\left((\ln w)_{x x}\right)^{2}+2(\ln w)_{x t} \\
2 \delta(\ln w)_{x x x} \\
0
\end{array}\right) .
\end{aligned}
$$

Noticing $(\ln w)_{x x}=\left((\ln w)_{x}\right)_{x}$, we have

$$
\mathbf{M}_{3} \mathbf{Z}_{t}^{*}+\mathbf{K}_{3} \mathbf{Z}_{x}^{*}=\mathbf{H}_{3}
$$

where

$$
\begin{aligned}
\mathbf{M}_{3} & =\left(\begin{array}{cccc}
0 & 0 & 0 & 0 \\
0 & 0 & 0 & 0 \\
0 & 0 & 0 & 0 \\
0 & 0 & 0 & 0
\end{array}\right), \quad \mathbf{K}_{3}=\left(\begin{array}{cccc}
0 & 0 & 0 & 0 \\
0 & 0 & -2 \delta & 0 \\
0 & 2 \delta & 0 & 0 \\
1 & 0 & 0 & 0
\end{array}\right), \\
\mathbf{H}_{3} & =\left(\begin{array}{c}
12 \delta\left((\ln w)_{x x}\right)^{2}+2(\ln w)_{x t} \\
2 \delta(\ln w)_{x x x} \\
(\ln w)_{x x}
\end{array}\right) .
\end{aligned}
$$

Noticing antisymmetry of state variable's coefficient matrix, we have

$$
\mathbf{M}_{4} \mathbf{Z}_{t}^{*}+\mathbf{K}_{4} \mathbf{Z}_{x}^{*}=\mathbf{H}_{4}
$$

where

$$
\begin{aligned}
\mathbf{M}_{4} & =\left(\begin{array}{cccc}
0 & 0 & 0 & 0 \\
0 & 0 & 0 & 0 \\
0 & 0 & 0 & 0 \\
0 & 0 & 0 & 0
\end{array}\right), \quad \mathbf{K}_{4}=\left(\begin{array}{cccc}
0 & 0 & 0 & -1 \\
0 & 0 & -2 \delta & 0 \\
0 & 2 \delta & 0 & 0 \\
1 & 0 & 0 & 0
\end{array}\right), \\
\mathbf{H}_{4} & =\left(\begin{array}{c}
-(\ln w)_{x x t} \\
12 \delta\left((\ln w)_{x x}\right)^{2}+2(\ln w)_{x t} \\
2 \delta(\ln w)_{x x x} \\
(\ln w)_{x x}
\end{array}\right)
\end{aligned}
$$


or

$$
\mathbf{M}_{5} \mathbf{Z}_{t}^{*}+\mathbf{K}_{5} \mathbf{Z}_{x}^{*}=\mathbf{H}_{5}
$$

where

$$
\begin{aligned}
\mathbf{M}_{5} & =\left(\begin{array}{cccc}
0 & 1 & 0 & 0 \\
0 & 0 & 0 & 0 \\
0 & 0 & 0 & 0 \\
0 & 0 & 0 & 0
\end{array}\right), \quad \mathbf{K}_{5}=\left(\begin{array}{cccc}
0 & 0 & 0 & -1 \\
0 & 0 & -2 \delta & 0 \\
0 & 2 \delta & 0 & 0 \\
1 & 0 & 0 & 0
\end{array}\right), \\
\mathbf{H}_{5} & =\left(\begin{array}{c}
12 \delta\left((\ln w)_{x x}\right)^{2}+2(\ln w)_{x t} \\
2 \delta(\ln w)_{x x x} \\
(\ln w)_{x x}
\end{array}\right) .
\end{aligned}
$$

Noticing antisymmetry of state variable's coefficient matrix, we have

$$
\mathbf{M Z}_{t}^{*}+\mathbf{K Z}_{x}^{*}=\mathbf{H}_{6}
$$

or

$$
\mathbf{M} \mathbf{Z}_{t}^{*}+\mathbf{K} \mathbf{Z}_{x}^{*}=\nabla_{\mathbf{Z}^{*}} S\left(\mathbf{Z}^{*}\right)
$$

where

$$
S\left(\mathbf{Z}^{*}\right)=4 \delta\left((\ln w)_{x x}\right)^{3}+(\ln w)_{x x}(\ln w)_{x t}+\delta\left((\ln w)_{x x x}\right)^{2}
$$

and

$$
\begin{aligned}
\mathbf{M} & =\left(\begin{array}{cccc}
0 & 1 & 0 & 0 \\
-1 & 0 & 0 & 0 \\
0 & 0 & 0 & 0 \\
0 & 0 & 0 & 0
\end{array}\right), \quad \mathbf{K}=\left(\begin{array}{cccc}
0 & 0 & 0 & -1 \\
0 & 0 & -2 \delta & 0 \\
0 & 2 \delta & 0 & 0 \\
1 & 0 & 0 & 0
\end{array}\right), \\
\mathbf{H}_{6} & =\left(\begin{array}{c}
12 \delta\left((\ln w)_{x x}\right)^{2}+(\ln w)_{x t} \\
2 \delta(\ln w)_{x x x} \\
(\ln w)_{x x}
\end{array}\right) .
\end{aligned}
$$

Multiplying $12 \delta$ to Eq. (41) and noticing $u=12 \delta(\ln w)_{x x}$, and introducing a state variable $\mathbf{Z}=12 \delta \mathbf{Z}^{*}=(\phi, u, v, \omega)^{\mathrm{T}}$, we have

$$
\left(\begin{array}{cccc}
0 & 1 & 0 & 0 \\
-1 & 0 & 0 & 0 \\
0 & 0 & 0 & 0 \\
0 & 0 & 0 & 0
\end{array}\right)\left(\begin{array}{c}
\phi \\
u \\
v \\
\omega
\end{array}\right)_{t}+\left(\begin{array}{cccc}
0 & 0 & 0 & -1 \\
0 & 0 & -2 \delta & 0 \\
0 & 2 \delta & 0 & 0 \\
1 & 0 & 0 & 0
\end{array}\right)\left(\begin{array}{c}
\phi \\
u \\
v \\
\omega
\end{array}\right)_{x}=\left(\begin{array}{c}
0 \\
u^{2}+\omega \\
2 \delta v \\
u
\end{array}\right)
$$


or

$$
\mathbf{M Z}_{t}+\mathbf{K Z}_{x}=\nabla_{\mathbf{Z}} S(\mathbf{Z})
$$

where $u=\phi_{x}, v=u_{x}, \omega=\phi_{t}$ and $S(\mathbf{Z})=\frac{u^{3}}{3}+\delta v^{2}+u \omega$.

Equation (42) is multi-symplectic structure of the KdV equation.

In summary, applying the homogeneous balance of undetermined coefficients method to the KdV equation and noticing the differential linearity with respect to $x, t$ on the lefthand side of Eq. (39), we get Eq. (40) which can inspire one how to define the state variable. Then considering antisymmetry of state variable's coefficient matrix and total differential of Hamiltonian function, Eq. (42) is naturally obtained.

Remark 5 Similar to the above process, let $f(y)=f(y(t))$ be a smooth function on $R, \alpha, \gamma$ $(\gamma \neq 0)$ are constants and $\ddot{y}=\frac{d^{2} y}{d t^{2}}$, the second-order ODE

$$
\alpha y+\int f(y) d y+\gamma \ddot{y}=0
$$

which has a compact form

$$
\mathbf{A} \dot{\mathbf{Y}}=\nabla_{\mathbf{Y}} H(\mathbf{Y})
$$

with $\mathbf{Y}=(\varphi, y, v, \rho)^{\mathrm{T}}, H(\mathbf{Y})=2 \iint f(y) d^{2} y+\rho y+\gamma v^{2}$ and

$$
\mathbf{A}=\left(\begin{array}{cccc}
0 & \alpha & 0 & -1 \\
-\alpha & 0 & -2 \gamma & 0 \\
0 & 2 \gamma & 0 & 0 \\
1 & 0 & 0 & 0
\end{array}\right)
$$

In fact, introducing $y=\dot{\varphi}, v=\dot{y}, \rho=\alpha y$, Eq. (43) can be stated as a system of first-order equations such that

$$
\begin{aligned}
& \alpha \dot{y}-\dot{\rho}=0, \\
& -\alpha \dot{\varphi}-2 \gamma \dot{v}=2 \int f(y) d y+\rho, \\
& 2 \gamma \dot{y}=2 \gamma \nu, \\
& \dot{\varphi}=y,
\end{aligned}
$$

which is equivalent to Eq. (44).

It is easily seen by noting that

$$
\frac{d}{d t} H(\mathbf{Y})=\left(\nabla_{\mathbf{Y}} H(\mathbf{Y})\right)^{\mathrm{T}} \dot{\mathbf{Y}}=-\dot{\mathbf{Y}}^{\mathrm{T}} \mathbf{A} \dot{\mathbf{Y}}=0
$$

because $\mathbf{A}$ is a skew-symmetric matrix. Thus, $H$ is constant along trajectories, and this implies conservation of total energy.

Assuming $\psi_{t}(\mathbf{Y})$ is the time $t$ flow map of Eq. (44), we get the variational equation

$$
\frac{d}{d t}\left(\partial_{\mathbf{Y}} \psi_{t}\right)=\mathbf{A}^{-1} H_{\mathbf{Y Y}} \partial_{\mathbf{Y}} \psi_{t},
$$


which implies

$$
\begin{aligned}
\frac{d}{d t}\left(\left(\partial_{\mathbf{Y}} \psi_{t}\right)^{\mathrm{T}} \mathbf{A}\left(\partial_{\mathbf{Y}} \psi_{t}\right)\right) & =\left(\frac{d}{d t}\left(\partial_{\mathbf{Y}} \psi_{t}\right)\right)^{\mathrm{T}} \mathbf{A}\left(\partial_{\mathbf{Y}} \psi_{t}\right)+\left(\partial_{\mathbf{Y}} \psi_{t}\right)^{\mathrm{T}} \mathbf{A}\left(\frac{d}{d t}\left(\partial_{\mathbf{Y}} \psi_{t}\right)\right) \\
& =\left(\partial_{\mathbf{Y}} \psi_{t}\right)^{\mathrm{T}} H_{\mathbf{Y} \mathbf{Y}} \mathbf{A}^{-\mathrm{T}} \mathbf{A}\left(\partial_{\mathbf{Y}} \psi_{t}\right)+\left(\partial_{\mathbf{Y}} \psi_{t}\right)^{\mathrm{T}} \mathbf{A}^{-1} H_{\mathbf{Y} \mathbf{Y}}\left(\partial_{\mathbf{Y}} \psi_{t}\right) \\
& =-\left(\partial_{\mathbf{Y}} \psi_{t}\right)^{\mathrm{T}} H_{\mathbf{Y} \mathbf{Y}}\left(\partial_{\mathbf{Y}} \psi_{t}\right)+\left(\partial_{\mathbf{Y}} \psi_{t}\right)^{\mathrm{T}} H_{\mathbf{Y} \mathbf{Y}}\left(\partial_{\mathbf{Y}} \psi_{t}\right)=0 .
\end{aligned}
$$

Equations (47) and (48) are similar to the properties of symplectic structure.

\section{Multi-symplectic structure of the generalized KdV-type equation}

In this section, the definition of generalized KdV-type equation and its multi-symplectic structure are given respectively.

Definition 2 Let $u, f$ and $g$ be smooth functions on $R$, PDE

$$
u_{t}+f(u) u_{x}+\left(g\left(u_{x}\right)\right)_{x x}=0,
$$

is called the generalized KdV-type equation.

When $f(u)=u$ and $g\left(u_{x}\right)=\delta u_{x}$, Eq. (49) is the KdV equation. When $f(u)=\alpha+\beta u^{\lambda}+\gamma u^{2 \lambda}$ and $g\left(u_{x}\right)=\delta u_{x}$, Eq. (49) is the generalized KdV-mKdV equation

$$
u_{t}+\left(\alpha+\beta u^{\lambda}+\gamma u^{2 \lambda}\right) u_{x}+\delta u_{x x x}=0
$$

where $\alpha, \beta, \gamma$ and $\lambda$ are known constants. Equation (50) has applications in a variety of areas such as fluid mechanics, quantum and crystal lattice theory. Obviously, the KdV equation and the KdV-mKdV equation are special cases of Eq. (49). Moreover, Eq. (49) has multi-symplectic structure. Now, we construct multi-symplectic structure of Eq. (49).

Similar to Section 2, setting $u=\phi_{x}$, Eq. (49) becomes

$$
\phi_{x t}+f\left(\phi_{x}\right) \phi_{x x}+\left(g\left(\phi_{x x}\right)\right)_{x x}=0 .
$$

Integrating the above equation with respect to $x$ once and setting integration constant to zero yield

$$
\phi_{t}+\int f\left(\phi_{x}\right) d \phi_{x}+\left(g\left(\phi_{x x}\right)\right)_{x}=0 .
$$

Introducing $u=\phi_{x}, v=u_{x}, \omega=\phi_{t}, \sigma=g\left(\phi_{x x}\right)=g(v)$, Eq. (52) can be stated as a system of first-order equations such that

$$
\begin{aligned}
& -\phi_{t}-2 \sigma_{x}=2 \int f(u) d u+\omega, \\
& u_{t}-\omega_{x}=0, \\
& \phi_{x}=u, \\
& 2 u_{x}=2 v, \\
& 0=2 \sigma-2 g(v) .
\end{aligned}
$$


This is equivalent to a multi-symplectic structure as follows:

$$
\mathbf{M Z}_{t}+\mathbf{K Z}_{x}=\nabla_{\mathbf{Z}} S(\mathbf{Z})
$$

where $\mathbf{Z}=(u, \phi, \omega, \sigma, v)^{\mathrm{T}}, S(\mathbf{Z})=u \omega+2 \sigma v-2 \int g(v) d v+2 \iint f(u) d^{2} u$ and

$$
\mathbf{M}=\left(\begin{array}{ccccc}
0 & -1 & 0 & 0 & 0 \\
1 & 0 & 0 & 0 & 0 \\
0 & 0 & 0 & 0 & 0 \\
0 & 0 & 0 & 0 & 0 \\
0 & 0 & 0 & 0 & 0
\end{array}\right), \quad \mathbf{K}=\left(\begin{array}{ccccc}
0 & 0 & 0 & -2 & 0 \\
0 & 0 & -1 & 0 & 0 \\
0 & 1 & 0 & 0 & 0 \\
2 & 0 & 0 & 0 & 0 \\
0 & 0 & 0 & 0 & 0
\end{array}\right)
$$

Taking $f(u)=u$ and $g(v)=g\left(u_{x}\right)=\delta u_{x}$, Eq. (54) is reduced to Eq. (42). Obviously, taking $f(u)=\alpha+\beta u^{\lambda}+\gamma u^{2 \lambda}$ and $g\left(u_{x}\right)=\delta u_{x}$, multi-symplectic structure of the generalized KdVtype equation is reduced to multi-symplectic structure of the generalized $\mathrm{KdV}$-mKdV equation.

Remark 6 From Eq. (51), by using Lagrangian density, covariant Legendre transformation and covariant Hamiltonian [13], one can also get Eq. (54). The process is more tedious than our method.

For a given NLPDE, once its multi-symplectic structure is obtained, some algorithms which show long-time numerical stability, high precision and preserve the intrinsic properties of NLPDE such as multi-symplectic Runge-Kutta collocation scheme, Euler box scheme, Preissmen box scheme, explicit midpoint scheme, spectral discretizations scheme, can be easily applied to NLPDE.

\section{Conclusions}

The homogeneous balance of undetermined coefficients method is successfully used to establish the exact solutions and multi-symplectic structure of NLPDEs. Bilinear equation or homogeneous equation, $N$-soliton solutions, traveling wave solutions and multisymplectic structure are obtained respectively by applying the proposed method to the $\mathrm{KdV}$ equation. Accordingly, the definition and multi-symplectic structure of the generalized KdV-type equation are given. Many well-known NLPDEs can be handled by this method. The performance of this method is found to be simple and efficient. The availability of computer systems like Maple facilitates the tedious algebraic calculations. The homogeneous balance of undetermined coefficients method is also a standard and computable method, which allows us to solve complicated and tedious algebraic calculations.

Competing interests

The authors declare that they have no competing interests. 


\section{Acknowledgements}

The research is supported by the National Natural Science Foundation of China (11372252, 11372253 and 11432010), the Fundamental Research Funds for the Central Universities (3102014JCQ01035).

Received: 31 March 2015 Accepted: 18 August 2015 Published online: 04 September 2015

\section{References}

1. Ablowitz, MJ, Clarkson, PA: Solitons, Nonlinear Evolution Equations and Inverse Scattering. Cambridge University Press, New York (1991)

2. $\mathrm{He}, \mathrm{JH}$ : An approximate solution technique depending on an artificial parameter: a special example. Commun. Nonlinear Sci. Numer. Simul. 3, 92-97 (1998)

3. Feng, ZS: The first-integral method to study the Burgers-Korteweg-de Vries equation. J. Phys. A, Math. Gen. 35 , 343-349 (2002)

4. Wang, $M L, L i, X Z$, Zhang, JL: The ( $\left.\frac{G^{\prime}}{G}\right)$-expansion method and travelling wave solutions of nonlinear evolution equations in mathematical physics. Phys. Lett. A 372, 417-423 (2008)

5. Zhang, $\mathrm{H}$ : New application of the ( $\left.\frac{G^{\prime}}{G}\right)$-expansion method. Commun. Nonlinear Sci. Numer. Simul. 14, 3220-3225 (2009)

6. Hirota, R: Exact solution of the Korteweg-de Vries equation for multiple collisions of solitons. Phys. Rev. Lett. 27, 1192-1194 (1971)

7. Hirota, R: The Direct Method in Soliton Theory. Cambridge University Press, Cambridge (2004)

8. Wang, ML: Solitary wave solutions for variant Boussinesq equations. Phys. Lett. A 199, 169-172 (1995)

9. Bai, CL: Extended homogeneous balance method and Lax pairs, Backlund transformation. Commun. Theor. Phys. 37, 645-648 (2002)

10. He, JH: An new approach to nonlinear partial differential equations. Commun. Nonlinear Sci. Numer. Simul. 2, 230-235 (1997)

11. Wazwaz, AM: The tanh method: exact solutions of the sine-Gordon and the sinh-Gordon equations. Appl. Math. Comput. 167, 1196-1201 (2005)

12. Khan, K, Akbar, MA, Rayhanul Islam, SM: Exact solutions for (1 + 1)-dimensional nonlinear dispersive modified Benjamin-Bona-Mahony equation and coupled Klein-Gordon equations. SpringerPlus 3, 724-731 (2014)

13. Wang, YS, Hong, JL: Multi-symplectic algorithms for Hamiltonian partial differential equations. Commun. Appl. Math. Comput. 27, 164-230 (2013)

14. Marsden, JE, Patrick, GP, Shkoller, S: Multi-symplectic geometry, variational integrations, and nonlinear PDEs. Commun. Math. Phys. 199, 351-395 (1999)

15. Bridges, TJ, Reich, S: Multi-symplectic integrators: numerical schemes for Hamiltonian PDEs that conserve symplecticity. Phys. Lett. A 284, 184-193 (2001)

16. Bridges, TJ, Reich, S: Numerical methods for Hamiltonian PDEs. J. Phys. A, Math. Gen. 39, 5287-5320 (2006)

17. Reich, S: Multi-symplectic Runge-Kutta collocation methods for Hamiltonian wave equations. J. Comput. Phys. 157, 473-499 (2000)

18. Moore, BE, Reich, S: Multi-symplectic integration methods for Hamiltonian PDEs. Future Gener. Comput. Syst. 19. 395-402 (2003)

19. Wang, YS, Wang, B, Qin, MZ: Numerical implementation of the multi-symplectic Perissman scheme and its equivalent schemes. Appl. Math. Comput. 149, 299-326 (2004)

20. Chen, JB: A multi-symplectic pseudospectral method for seismic modeling. Appl. Math. Comput. 186, 1612-1616 (2007)

21. Hu, WP, Deng, ZC, Han, SM: An implicit difference scheme focusing on the local conservation properties for Burgers equation. Int. J. Comput. Methods 9, 1240028 (2012)

22. Hu, WP, Deng, ZC, Han, SM, Zhang, WR: Generalized multi-symplectic integrators for a class of Hamiltonian nonlinear wave PDEs. J. Comput. Phys. 235, 394-406 (2013)

23. Hu, WP, Deng, ZC, Qin, YY, Zhang, WR: Multi-symplectic method for the generalized $(2+1)$-dimensional KdV-mKdV equation. Acta Mech. Sin. 28, 793-800 (2012)

24. Hu, WP, Deng, ZC: Multi-symplectic method for the generalized fifth order KdV equation. Chin. Phys. B 17, $3923-3929$ (2008)

\section{Submit your manuscript to a SpringerOpen ${ }^{\circ}$ journal and benefit from:}

- Convenient online submission

- Rigorous peer review

- Immediate publication on acceptance

- Open access: articles freely available online

- High visibility within the field

- Retaining the copyright to your article 